\title{
Mixed boundary value problems of diffraction by a half-plane with an obstacle perpendicular to the boundary $^{1}$
}

\author{
L.P. Castro*2, D. Kapanadze ${ }^{\sharp,}$ \\ * CIDMA \& Department of Mathematics, University of Aveiro, Portugal \\ \# A. Razmadze Mathematical Institute, Tbilisi State University, Georgia
}

\begin{abstract}
The paper is devoted to the analysis of wave diffraction problems modelled by classes of mixed boundary conditions and the Helmholtz equation, within a half-plane with a crack. Potential theory together with Fredholm theory, and explicit operator relations, are conveniently implemented to perform the analysis of the problems. In particular, an interplay between Wiener-Hopf plus/minus Hankel operators and Wiener-Hopf operators assumes a relevant preponderance in the final results. As main conclusions, this study reveals conditions for the well-posedness of the corresponding boundary value problems in certain Sobolev spaces and equivalent reduction to systems of Wiener-Hopf equations.
\end{abstract}

2010 MSC: 35J05; 35B30; 35C15; 35J25; 35P25; 47A20; 47A53; 47A68; 47B35; 47G30; 78A45.

Keywords: Helmholtz equation; wave diffraction; boundary value problems; potential method; oscillating symbols.

\section{Introduction}

We use potential theory and Fredholm theory to analyse wave diffraction problems modelled by the Helmholtz equation in a half-plane interrupted by a perpendicular crack, when considering different classes of Dirichlet and

\footnotetext{
${ }^{1}$ This is the accepted version of the following article: L.P. Castro, D. Kapanadze, Mixed boundary value problems of diffraction by a half-plane with an obstacle perpendicular to the boundary, Mathematical Methods in the Applied Sciences 37 (2014), 1412-1427, which has been published in final form at http://onlinelibrary.wiley.com/doi/10.1002/mma.2900/pdf. This article may be used for non-commercial purposes in accordance with the Wiley Self-Archiving Policy http://olabout.wiley.com/WileyCDA/Section/id-828039.html.

${ }^{2}$ Corresponding author.
} 
Neumann boundary conditions. The problems are reformulated as boundary integral systems which are firstly analysed in view of obtaining conditions for the existence and uniqueness of solutions within a scale of Bessel potential spaces. Several recent works could be referred as being devoted to present a mathematical analysis of wave diffraction problems governed by the Helmholtz equation and which are somehow natural physical generalizations of the original works of Sommerfeld (see [6]-[11], [17, 19, 22], [24]-[41] and $[43,44])$.

One important feature of the present work is that we will be able to explicitly identify the Fourier symbols of the pseudodifferential operators which are deduced from the problems. In particular, this allows the possibility of having representation formulas for the solutions. Interactions with the classical Wiener-Hopf technique can also be here recognized, where a crucial step in the method consists in decomposing the so-called Fourier symbol into a product of two factors with certain analyticity properties. Anyway, in the present work, we will face semi-almost periodic Fourier symbols for which corresponding factorizations in the appropriate sense are - in general - not trivial. Moreover, due to the complexity of the boundary value problems, the method we propose to deal with the problems do not directly lead to a Wiener-Hopf operator but instead to somehow coupled Wiener-Hopf plus Hankel and Wiener-Hopf minus Hankel operators. Then, a convenient procedure of explicit operator relations will allow us to find out some associated matrix Wiener-Hopf equations and to derive the final conclusions based on certain factorizations of associated matrix functions.

In the following section, we start by introducing some auxiliary notation and present the mathematical formulation of the problems. In section 3, a uniqueness result (for the solutions) is obtained for all the problems in consideration. Then, in section 4, we revise the properties of some integral operators and consequent results from potential theory. Section 5 is devoted to equivalently reformulate the problems under analysis by means of integral equations characterized by Wiener-Hopf plus and minus Hankel operators. Then, in section 6 , we will consider explicit operator relations for those operators - which will help us to derive their Fredholm properties later on. In section 7 we will analyse the Fredholm properties of certain Wiener-Hopf operators somehow related with the previously obtained operators. These Wiener-Hopf operators will be characterized by certain Fourier symbols from the $C^{*}$-algebra of the semi-almost periodic four by four matrix functions on the real line. Finally, in the last section, the main conclusion on 
the existence, uniqueness and integral representations of the solutions of the problems are assembled in a unique result. We would like to point out that the present paper is a continuation of the author's work begun in $[6,7,8,9]$ and that an alternative approach can be based upon the articles [18, 35]. The present approach has some advantages since e.g., in some cases, it also allows the consideration of pure (non complex) real wave numbers [8] but this will not be the case in the present work.

\section{Mathematical formulation of the problems}

In this section we will present the formulation of our problems from the mathematical point of view. To this end, we start by introducing some useful general notation. As usual, $\mathcal{S}\left(\mathbb{R}^{n}\right)$ denotes the Schwartz space of all rapidly decreasing functions and $\mathcal{S}^{\prime}\left(\mathbb{R}^{n}\right)$ the dual space of tempered distributions on $\mathbb{R}^{n}$. The Bessel potential space $H^{s}\left(\mathbb{R}^{n}\right)$, with $s \in \mathbb{R}$, is formed by the elements $\varphi \in \mathcal{S}^{\prime}\left(\mathbb{R}^{n}\right)$ such that $\|\varphi\|_{H^{s}\left(\mathbb{R}^{n}\right)}=\left\|\mathcal{F}^{-1}\left(1+|\xi|^{2}\right)^{s / 2} \cdot \mathcal{F} \varphi\right\|_{L_{2}\left(\mathbb{R}^{n}\right)}$ is finite. As the notation indicates, $\|\cdot\|_{H^{s}\left(\mathbb{R}^{n}\right)}$ is a norm for the space $H^{s}\left(\mathbb{R}^{n}\right)$ which makes it a Banach space. Here, $\mathcal{F}=\mathcal{F}_{x \mapsto \xi}$ denotes the Fourier transformation in $\mathbb{R}^{n}$.

For a given Lipschitz domain $\mathcal{D}$, on $\mathbb{R}^{n}$, we denote by $\widetilde{H}^{s}(\mathcal{D})$ the closed subspace of $H^{s}\left(\mathbb{R}^{n}\right)$ whose elements have supports in $\overline{\mathcal{D}}$, and $H^{s}(\mathcal{D})$ denotes the space of generalized functions on $\mathcal{D}$ which have extensions into $\mathbb{R}^{n}$ that belong to $H^{s}\left(\mathbb{R}^{n}\right)$. The space $\widetilde{H}^{s}(\mathcal{D})$ is endowed with the subspace topology, and on $H^{s}(\mathcal{D})$ we introduce the norm of the quotient space $H^{s}\left(\mathbb{R}^{n}\right) / \widetilde{H}^{s}\left(\mathbb{R}^{n} \backslash \overline{\mathcal{D}}\right)$. Throughout the paper we will use the notation $\mathbb{R}_{ \pm}^{n}:=$ $\left\{x=\left(x_{1}, \ldots, x_{n-1}, x_{n}\right) \in \mathbb{R}^{n}: \pm x_{n}>0\right\}$. Note that the spaces $H^{0}\left(\mathbb{R}_{+}^{n}\right)$ and $\widetilde{H}^{0}\left(\mathbb{R}_{+}^{n}\right)$ can be identified, and we will denote them by $L_{2}\left(\mathbb{R}_{+}^{n}\right)$. For a comprehensive treatment of Sobolev spaces we refer to [1], for unbounded Lipschitz domains see also [34], and for domains with conical points, edges, polyhedra, cuts (or cracks), slits or holes we cite [20].

Let $\Omega:=\left\{\left(x_{1}, x_{2}\right) \in \mathbb{R}^{2}: x_{1}>0, x_{2} \in \mathbb{R}\right\}, \Gamma_{1}:=\left\{\left(x_{1}, 0\right): x_{1} \in \mathbb{R}\right\}$, and $\Gamma_{2}:=\left\{\left(0, x_{2}\right): x_{2} \in \mathbb{R}\right\}$. Let further $\mathcal{C}:=\left\{\left(x_{1}, 0\right): 0<x_{1}<a\right\} \subset \Gamma_{1}$ for a certain positive number $a$ and $\Omega_{\mathcal{C}}:=\Omega \backslash \overline{\mathcal{C}}$. Clearly, $\partial \Omega=\Gamma_{2}$ and $\partial \Omega_{\mathcal{C}}=\Gamma_{2} \cup \overline{\mathcal{C}}$.

For our purposes below we introduce further notations: $\Omega_{1}:=\left\{\left(x_{1}, x_{2}\right) \in\right.$ $\left.\mathbb{R}^{2}: x_{1}>0, x_{2}>0\right\}$ and $\Omega_{2}:=\left\{\left(x_{1}, x_{2}\right) \in \mathbb{R}^{2}: x_{1}>0, x_{2}<0\right\}$, then $\partial \Omega_{j}=\mathcal{S}_{j} \cup \mathcal{S}$, for $j=1,2$, where $\mathcal{S}:=\left\{\left(x_{1}, 0\right): x_{1} \geq 0\right\} \subset \Gamma_{1}, \mathcal{S}_{1}:=\left\{\left(0, x_{2}\right)\right.$ : 
$\left.x_{2} \geq 0\right\} \subset \Gamma_{2}$, and $\mathcal{S}_{2}:=\left\{\left(0, x_{2}\right): x_{2} \leq 0\right\} \subset \Gamma_{2}$. Finally, we introduce the following unit normal vectors $n_{1}=\overrightarrow{(0,-1)}$ on $\Gamma_{1}$ and $n_{2}=\overrightarrow{(-1,0)}$ on $\Gamma_{2}$.

Let $\varepsilon \in\left[0, \frac{1}{2}\right)$. We are interested in studying the problem of existence and uniqueness of an element $u \in H^{1+\varepsilon}\left(\Omega_{\mathcal{C}}\right)$, such that

$$
\left(\Delta+k^{2}\right) u=0 \text { in } \Omega_{\mathcal{C}},
$$

and $u$ satisfies one of the following four representative mixed boundary conditions:

$$
\begin{aligned}
& {[u]_{\mathcal{C}}^{+}=g_{0}^{+}, \quad\left[\partial_{n_{1}} u\right]_{\mathcal{C}}^{-}=g_{1}^{-} \quad \text { on } \quad \mathcal{C}, \quad \text { and } \quad[u]_{\mathcal{S}_{j}}^{+}=h_{j} \quad \text { on } \quad \mathcal{S}_{j},(2.2)} \\
& {[u]_{\mathcal{C}}^{+}=g_{0}^{+}, \quad\left[\partial_{n_{1}} u\right]_{\mathcal{C}}^{-}=g_{1}^{-} \quad \text { on } \quad \mathcal{C}, \quad \text { and } \quad\left[\partial_{n_{2}} u\right]_{\mathcal{S}_{j}}^{+}=f_{j} \quad \text { on } \quad \mathcal{S}_{j},(2.3)} \\
& \left\{\begin{array} { l } 
{ [ u ] _ { \mathcal { C } } ^ { + } = g _ { 0 } ^ { + } \text { on } \mathcal { C } , } \\
{ [ \partial _ { n _ { 1 } } u ] _ { \mathcal { C } } ^ { - } = g _ { 1 } ^ { - } \text { on } \mathcal { C } , }
\end{array} \quad \text { and } \quad \left\{\begin{array}{l}
{\left[\partial_{n_{2}} u\right]_{\mathcal{S}_{1}}^{+}=f_{1} \text { on } \mathcal{S}_{1},} \\
{[u]_{\mathcal{S}_{2}}^{+}=h_{2} \text { on } \mathcal{S}_{2},}
\end{array}\right.\right. \\
& \left\{\begin{array} { l } 
{ [ u ] _ { \mathcal { C } } ^ { + } = g _ { 0 } ^ { + } \text { on } \mathcal { C } , } \\
{ [ \partial _ { n _ { 1 } } u ] _ { \mathcal { C } } ^ { - } = g _ { 1 } ^ { - } \text { on } \mathcal { C } , }
\end{array} \quad \text { and } \quad \left\{\begin{array}{l}
{[u]_{\mathcal{S}_{1}}^{+}=h_{1} \text { on } \mathcal{S}_{1},} \\
{\left[\partial_{n_{2}} u\right]_{\mathcal{S}_{2}}^{+}=f_{2} \text { on } \mathcal{S}_{2},}
\end{array}\right.\right.
\end{aligned}
$$

for $j=1,2$. Here the wave number $k \in \mathbb{C} \backslash \mathbb{R}$ is given. The elements $[u]_{\mathcal{S}_{j}}^{+}$ and $\left[\partial_{n_{2}} u\right]_{\mathcal{S}_{j}}^{+}$denote the Dirichlet and the Neumann traces on $\mathcal{S}_{j}$, respectively, while by $[u]_{\mathcal{C}}^{ \pm}$we denote the Dirichlet traces on $\mathcal{C}$ from both sides of the screen and by $\left[\partial_{n_{1}} u\right]_{\mathcal{C}}^{ \pm}$we denote the Neumann traces on $\mathcal{C}$ from both sides of the crack.

Throughout the paper on the given data we assume that $h_{j} \in H^{1 / 2+\varepsilon}\left(\mathcal{S}_{j}\right)$, $f_{j} \in H^{-1 / 2+\varepsilon}\left(\mathcal{S}_{j}\right)$, for $j=1,2$, and $g_{j}^{ \pm} \in H^{1 / 2-i+\varepsilon}(\mathcal{C})$, for $j=0,1$. Furthermore, we suppose that they satisfy the following compatibility conditions:

$$
\begin{gathered}
\chi_{0}\left(g_{0}^{+}-r_{\mathcal{C}} h_{1} \circ e^{i \frac{\pi}{2}}\right) \in r_{\mathcal{C}} \widetilde{H}^{1 / 2+\varepsilon}(\mathcal{C}), \\
\chi_{0}\left(g_{1}^{-}-r_{\mathcal{C}} f_{2} \circ e^{-i \frac{\pi}{2}}\right) \in r_{\mathcal{C}} \widetilde{H}^{-1 / 2+\varepsilon}(\mathcal{C}) .
\end{gathered}
$$

Here, $r_{\mathcal{C}}$ denotes the restriction operator to $\mathcal{C}$ and $\chi_{0} \in C^{\infty}([0, a])$ is such that $\chi_{0}(x) \equiv 1$ for $x \in[0, a / 3]$ and $\chi_{0}(x) \equiv 0$ for $x \in[2 a / 3, a]$.

From now on we will refer to:

- Problem $\mathcal{P}_{\text {mixed-D }}$ as the problem characterized by (2.1), (2.2), and (2.6); 
- Problem $\mathcal{P}_{\text {mixed }-N}$ as the one characterized by (2.1), (2.3), (2.7);

- Problem $\mathcal{P}_{\text {mixed }}$ as the one characterized by (2.1) and (2.4);

- Problem $\mathcal{P}_{\text {mixed }}$ as the one characterized by (2.1), (2.5), (2.6), and (2.7).

As about the just stated compatibility conditions, note that they are necessary conditions for the well-posedness of the corresponding problems. Note also that, the compatibility condition (2.7) included in Problems $\mathcal{P}_{\text {mixed }-N}$ and $\mathcal{P}_{\text {mixed }}$ is additional restrictions only for $\varepsilon=0$.

\section{Uniqueness of solutions}

The uniqueness result for the solutions of problems in consideration is exhibited in this section.

Theorem 3.1. Each one of the problems $\mathcal{P}_{\text {mixed-D }}, \mathcal{P}_{\text {mixed-N }}, \mathcal{P}_{\text {mixed }}$, and $\mathcal{P}_{\text {mixed }}$ has at most one solution.

Proof. This proof may be considered standard and is based on the use of the Green formula in convenient domains. It is sufficient to consider the case $\varepsilon=0$.

Let $R$ be a sufficiently large positive number and $B(R)$ be the disk centered at the origin with radius $R$. Set $\Omega_{R}:=\Omega_{\mathcal{C}} \cap B(R)$. Note that the domain $\Omega_{R}$ has a piecewise smooth boundary $S_{R}$ including both sides of $\mathcal{C}$ and denote by $n(x)$ the outward unit normal vector at the non-singular points $x \in S_{R}$.

We will assume $u$ to be a solution of the homogeneous problem. Then, the first Green identity for $u$ and its complex conjugate $\bar{u}$ in the domain $\Omega_{R}$, together with zero boundary conditions on $S_{R}$ yields

$$
\int_{\Omega_{R}}\left[|\nabla u|^{2}-k^{2}|u|^{2}\right] d x=\int_{\partial B(R) \cap \Omega}\left(\partial_{n} u\right) \bar{u} d S_{R} .
$$

Note that, since $\Im \mathrm{m} k \neq 0$, the integral $\int_{\partial B(R) \cap \Omega}\left(\partial_{n} u\right) \bar{u} d S$ tends to zero as $R \rightarrow \infty$. Indeed, in $(R, \phi)$ polar coordinates we have

$$
\int_{\partial B(R) \cap \Omega}\left(\partial_{n} u\right) \bar{u} d S=R \int_{-\frac{\pi}{2}}^{\frac{\pi}{2}}\left(\partial_{n} u\right) \bar{u} d \phi=R \lim _{\delta_{1}, \delta_{2} \rightarrow 0+} \int_{\delta_{1}-\frac{\pi}{2}}^{\frac{\pi}{2}-\delta_{2}}\left(\partial_{n} u\right) \bar{u} d \phi
$$


and we take into account that the solution $u \in H^{1}(\Omega)$ of the Helmholtz equation exponentially decays at infinity in the corresponding sector (which follows from the representation formula of a solution of the Helmholtz equation; cf. [45]). Therefore, passing to the limit as $R \rightarrow \infty$ in (3.1), it follows

$$
\int_{\Omega_{\mathcal{C}}}\left[|\nabla u|^{2}-k^{2}|u|^{2}\right] d x=0 .
$$

From the real and imaginary parts of the last identity, we obtain

$$
\left\{\begin{array}{l}
\int_{\Omega_{\mathcal{C}}}\left[|\nabla u|^{2}+\left((\Im \mathrm{m} k)^{2}-(\Re \mathrm{e} k)^{2}\right)|u|^{2}\right] d x=0, \\
-2(\Re \mathrm{e} k)(\Im \mathrm{m} k) \int_{\Omega_{\mathcal{C}}}|u|^{2} d x=0 .
\end{array}\right.
$$

Thus, from the condition $\Im \mathrm{m} k \neq 0$, it follows from (3.2) that $u=0$ in $\Omega_{\mathcal{C}}$

\section{Potential theory}

In the present section, we will recall some results from potential theory (as well as some of their interactions with even and odd extension operators) which will be useful in our future reasoning.

From now on, throughout the remaining part of the paper, and without lost of generality, we assume that $\Im \mathrm{m} k>0$ (since the complementary case $\Im \mathrm{m} k<0$ runs with obvious changes).

Let us denote the standard fundamental solution of the Helmholtz equation (in two dimensions) by

$$
\mathcal{K}(x):=-\frac{i}{4} H_{0}^{(1)}(k|x|),
$$

where $H_{0}^{(1)}(k|x|)$ is the Hankel function of the first kind of order zero (cf. [19, $\S 3.4]$ or $[22, \S 2.1])$. Furthermore, we introduce the single and double layer potentials on $\Gamma_{j}$ :

$$
\begin{aligned}
V_{j}(\psi)(x) & =\int_{\Gamma_{j}} \mathcal{K}(x-y) \psi(y) d_{y} \Gamma_{j}, \quad x \notin \Gamma_{j}, \\
W_{j}(\varphi)(x) & =\int_{\Gamma_{j}}\left[\partial_{n_{j}(y)} \mathcal{K}(x-y)\right] \varphi(y) d_{y} \Gamma_{j}, \quad x \notin \Gamma_{j},
\end{aligned}
$$


where $j=1,2$ and $\psi, \varphi$ are density functions. Note that for $j=1$ sometimes we will write $\mathbb{R}$ instead of $\Gamma_{1}$. In this case, for example, the single layer potential defined above has the form

$$
V_{1}(\psi)\left(x_{1}, x_{2}\right)=\int_{\mathbb{R}} \mathcal{K}\left(x_{1}-y, x_{2}\right) \psi(y) d y, \quad x_{2} \neq 0 .
$$

Set $\mathbb{R}_{ \pm}^{2}:=\left\{\left(x_{1}, x_{2}\right) \in \mathbb{R}^{2}: x_{2} \gtrless 0\right\}$ and let us first consider the operators $V:=V_{1}$ and $W:=W_{1}$.

Theorem 4.1 ([7]). The single and double layer potentials $V$ and $W$ are continuous operators

$$
V: H^{s}(\mathbb{R}) \rightarrow H^{s+1+\frac{1}{2}}\left(\mathbb{R}_{ \pm}^{2}\right), \quad W: H^{s+1}(\mathbb{R}) \rightarrow H^{s+1+\frac{1}{2}}\left(\mathbb{R}_{ \pm}^{2}\right),
$$

for all $s \in \mathbb{R}$.

Clearly, a similar result holds true for the operators $V_{2}$ and $W_{2}$.

Let us now recall some properties of the above introduced potentials. The following limit relations are well-known (cf. [7]):

$$
\begin{aligned}
& {[V(\psi)]_{\mathbb{R}}^{+}=[V(\psi)]_{\mathbb{R}}^{-}=: \mathcal{H}(\psi), \quad\left[\partial_{n} V(\psi)\right]_{\mathbb{R}}^{ \pm}=:\left[\mp \frac{1}{2} I\right](\psi),} \\
& {[W(\varphi)]_{\mathbb{R}}^{ \pm}=:\left[ \pm \frac{1}{2} I\right](\varphi), \quad\left[\partial_{n} W(\varphi)\right]_{\mathbb{R}}^{+}=\left[\partial_{n} W(\varphi)\right]_{\mathbb{R}}^{-}=: \mathcal{L}(\varphi),}
\end{aligned}
$$

where

$$
\begin{aligned}
\mathcal{H}(\psi)(z) & :=\int_{\mathbb{R}} \mathcal{K}(z-y) \psi(y) d y, \quad z \in \mathbb{R}, \\
\mathcal{L}(\varphi)(z) & :=\lim _{\mathbb{R}_{+}^{2} \ni x \rightarrow z \in \mathbb{R}} \partial_{n(x)} \int_{\mathbb{R}}\left[\partial_{n(y)} \mathcal{K}(y-x)\right] \varphi(y) d y, \quad z \in \mathbb{R},
\end{aligned}
$$

and $I$ denotes the identity operator.

In our further reasoning we will make a convenient use of the even and odd extension operators defined by $\ell^{e} \varphi(y)=\left\{\begin{array}{ll}\varphi(y), & y \in \mathbb{R}_{ \pm} \\ \varphi(-y), & y \in \mathbb{R}_{\mp}\end{array} \quad\right.$ and $\quad \ell^{o} \varphi(y)=\left\{\begin{array}{ll}\varphi(y), & y \in \mathbb{R}_{ \pm} \\ -\varphi(-y), & y \in \mathbb{R}_{\mp}\end{array}\right.$, respectively. 
Remark 4.2 ([17]). The following operators

$$
\begin{array}{ll}
\ell^{e}: H^{\varepsilon+\frac{1}{2}}\left(\mathbb{R}_{ \pm}\right) \longrightarrow H^{\varepsilon+\frac{1}{2}}(\mathbb{R}), & \ell^{o}: r_{\mathbb{R}_{ \pm}} \widetilde{H}^{\varepsilon+\frac{1}{2}}\left(\mathbb{R}_{ \pm}\right) \longrightarrow H^{\varepsilon+\frac{1}{2}}(\mathbb{R}), \\
\ell^{o}: H^{\varepsilon-\frac{1}{2}}\left(\mathbb{R}_{ \pm}\right) \longrightarrow H^{\varepsilon-\frac{1}{2}}(\mathbb{R}), & \ell^{e}: r_{\mathbb{R}_{ \pm}} \widetilde{H}^{\varepsilon-\frac{1}{2}}\left(\mathbb{R}_{ \pm}\right) \longrightarrow H^{\varepsilon-\frac{1}{2}}(\mathbb{R}),
\end{array}
$$

are continuous for all $\varepsilon \in[0,1 / 2)$.

Lemma 4.3 ([7]). If $0 \leq \varepsilon<1 / 2$, then

$$
\begin{aligned}
& r_{\Gamma_{2}} \circ V \circ \ell^{o} \psi=0, \quad r_{\Gamma_{2}} \circ W \circ \ell^{o} \tilde{\varphi}=0, \\
& r_{\Gamma_{2}} \circ \partial_{n_{2}} V \circ \ell^{e} \tilde{\psi}=0, \quad r_{\Gamma_{2}} \circ \partial_{n_{2}} W \circ \ell^{e} \varphi=0
\end{aligned}
$$

for all $\psi \in H^{\varepsilon-\frac{1}{2}}(\mathcal{S}), \tilde{\psi} \in r_{\mathcal{S}} \widetilde{H}^{\varepsilon-\frac{1}{2}}(\mathcal{S}), \varphi \in H^{\varepsilon+\frac{1}{2}}(\mathcal{S})$, and $\tilde{\varphi} \in r_{\mathcal{S}} \widetilde{H}^{\varepsilon+\frac{1}{2}}(\mathcal{S})$.

We would like to point out that analogous results are valid for the operators $V_{2}$ and $W_{2}$.

\section{The Wiener-Hopf plus/minus Hankel oper- ators associated with the problems}

The main goal of the present section is to equivalently reformulate the problems under analysis by means of integral equations characterized by WienerHopf plus and minus Hankel operators. To this end, we will make a convenient use of the pseudodifferential operators introduced in the last section together with some auxiliary operators based on the odd and even extension operators and the reflection operator $J$, defined by the rule

$$
J \psi(y)=\psi(-y) \quad \text { for all } \quad y \in \mathbb{R} .
$$

\subsection{The mixed-D problem}

The boundary value problem $\mathcal{P}_{\text {mixed }-D}$ can be equivalently rewritten [38] in the following form: Find $u_{j} \in H^{1+\varepsilon}\left(\Omega_{j}\right), j=1,2$ such that

$$
\begin{array}{rrr}
\left(\Delta+k^{2}\right) u_{j}=0 & \text { in } & \Omega_{j}, \\
{\left[u_{j}\right]_{\mathcal{S}_{j}}^{+}=h_{j}} & \text { on } & \mathcal{S}_{j}, \\
{\left[u_{1}\right]_{\mathcal{C}}^{+}=g_{0}^{+}, \quad\left[\partial_{n_{1}} u_{2}\right]_{\mathcal{C}}^{-}=g_{1}^{-}} & \text {on } & \mathcal{C},
\end{array}
$$


and

$$
\left[u_{1}\right]_{\mathcal{C}^{c}}^{+}-\left[u_{2}\right]_{\mathcal{C}^{c}}^{-}=0, \quad\left[\partial_{n_{1}} u_{1}\right]_{\mathcal{C}^{c}}^{+}-\left[\partial_{n_{1}} u_{2}\right]_{\mathcal{C}^{c}}^{-}=0 \quad \text { on } \quad \mathcal{C}^{c}
$$

where $\mathcal{C}^{c}=\mathcal{S} \backslash \overline{\mathcal{C}}$, and assuming the compatibility condition (2.6).

Proposition 5.1. A function $u \in H^{1+\varepsilon}\left(\Omega_{\mathcal{C}}\right)$ is a solution of Problem $\mathcal{P}_{\text {mixed }-D}$ if and only if $u$ can be represented by

$$
u_{1}=2 W_{1}\left(\ell^{o}\left(\ell_{+} g_{0}^{+}-\left[2 W_{2}\left(\ell^{e} h_{1}\right)\right]_{\mathcal{S}}^{+}\right)+\ell^{o} r_{\mathcal{S}} \varphi_{0}\right)+2 W_{2}\left(\ell^{e} h_{1}\right) \quad \text { in } \Omega_{1}
$$

and

$$
u_{2}=2 V_{1}\left(\ell^{o}\left(\ell_{+} g_{1}^{-}+r_{\mathcal{S}} \varphi_{1}\right)\right)+2 W_{2}\left(\ell^{e} h_{2}\right) \quad \text { in } \Omega_{2},
$$

where $\varphi_{0}$ and $\varphi_{1}$ are suitable elements of the spaces $\widetilde{H}^{\frac{1}{2}+\varepsilon}\left(\mathcal{C}^{c}\right)$ and $\widetilde{H}^{-\frac{1}{2}+\varepsilon}\left(\mathcal{C}^{c}\right)$, respectively, while $\ell_{+} g_{0}^{+} \in H^{\frac{1}{2}+\varepsilon}(\mathcal{S})$ is any fixed extension of $g_{0}^{+} \in H^{\frac{1}{2}+\varepsilon}(\mathcal{C})$ and $\ell_{+} g_{1}^{-} \in H^{-\frac{1}{2}+\varepsilon}(\mathcal{S})$ is any fixed extension of $g_{1}^{+} \in H^{-\frac{1}{2}+\varepsilon}(\mathcal{C})$.

Proof. From Theorem 3.1 we know already that Problem $\mathcal{P}_{\text {mixed-D }}$ has at most one solution. Thus, we need only to prove that $u_{j} \in H^{1+\varepsilon}\left(\Omega_{j}\right), j=1,2$, presented in (5.5)-(5.6) are solutions of (5.1)-(5.4) when considering the compatibility condition (2.6).

We stress that the compatibility condition (2.6) ensures us that

$$
\ell_{+} g_{0}^{+}-\left[2 W_{2}\left(\ell^{e} h_{1}\right)\right]_{\mathcal{S}}^{+} \in r_{\mathcal{S}} \widetilde{H}^{\frac{1}{2}+\varepsilon}(\mathcal{S})
$$

and, therefore, we may apply the first extension operator $\ell^{o}$ in the construction (5.5) of $u_{1}$. Here, it is also crucial to have $\varepsilon \in\left[0, \frac{1}{2}\right)$.

Using the results from Section 4 it is direct to verify that $u_{j}$ belong to the spaces $H^{1+\varepsilon}\left(\Omega_{j}\right)$ and satisfy equations (5.1)-(5.3). Therefore, it remains to satisfy the conditions (5.4) which together with (5.5) and (5.6) lead us to the following equation

$$
r_{\mathcal{C}^{c}} \mathcal{K} \ell^{o} r_{\mathcal{S}} \Upsilon=F^{D}
$$

where

$$
\mathcal{K}:=\left(\begin{array}{cc}
1 & 2 \mathcal{H} \\
2 \mathcal{L} & 1
\end{array}\right), \quad \Upsilon:=\left(\begin{array}{c}
\varphi_{0} \\
-\varphi_{1}
\end{array}\right)
$$

and $F^{D}:=\left(F_{0}^{D}, F_{1}^{D}\right)^{\top}$ is a known vector function, namely, we have

$$
F_{0}^{D}:=r_{\mathcal{C}^{c}}\left(2 \mathcal{H}\left(\ell^{o}\left(\ell_{+} g_{1}^{-}\right)\right)+\left[2 W_{2}\left(\ell^{e} h_{2}\right)\right]_{\mathcal{S}}^{-}-\ell_{+} g_{0}^{+}\right) \in H^{\frac{1}{2}+\varepsilon}\left(\mathcal{C}^{c}\right)
$$


and

$$
F_{1}^{D}:=r_{\mathcal{C}^{c}}\left(\ell_{+} g_{1}^{-}-2 \mathcal{L}\left(\ell^{o}\left(\ell_{+} g_{0}^{+}-\left[2 W_{2}\left(\ell^{e} h_{1}\right)\right]_{\mathcal{S}}^{+}\right)\right) \in H^{-\frac{1}{2}+\varepsilon}\left(\mathcal{C}^{c}\right) .\right.
$$

Here, we would like to point out that to obtain the just presented final form of $F_{1}^{D}$ we have used Lemma 4.3 for the operator $W_{2}$, and that the operator in (5.7) is defined in the appropriate sense:

$$
r_{\mathcal{C}^{c}} \mathcal{K} \ell^{o} r_{\mathcal{S}}: \begin{gathered}
\widetilde{H}^{\frac{1}{2}+\varepsilon}\left(\mathcal{C}^{c}\right) \\
\widetilde{H}^{-\frac{1}{2}+\varepsilon}\left(\mathcal{C}^{c}\right)
\end{gathered} \longrightarrow \begin{gathered}
H^{\frac{1}{2}+\varepsilon}\left(\mathcal{C}^{c}\right) \\
H^{-\frac{1}{2}+\varepsilon}\left(\mathcal{C}^{c}\right)
\end{gathered} .
$$

As a consequence of the equation (5.7) just derived, in view to obtain more information on the elements $\varphi_{0}$ and $\varphi_{1}$, we need to investigate the invertibility of the operator (5.9).

With the help of the operator $J$ and the shift convolution operators

$$
\operatorname{Op}\left(\tau_{ \pm a}\right):=\mathcal{F}^{-1} \tau_{ \pm a} \cdot \mathcal{F}
$$

(where we recall that $\mathcal{F}$ denotes the Fourier transformation and $\tau_{b}(\xi):=e^{i b \xi}$, $\xi \in \mathbb{R}$ ), we equivalently reduce the problem to the invertibility of the operator

$$
r_{\mathbb{R}_{+}} \mathcal{K}_{--}: \begin{gathered}
\tilde{H}^{\frac{1}{2}+\varepsilon}\left(\mathbb{R}_{+}\right) \\
\oplus \\
\tilde{H}^{-\frac{1}{2}+\varepsilon}\left(\mathbb{R}_{+}\right)
\end{gathered} \longrightarrow \begin{gathered}
H^{\frac{1}{2}+\varepsilon}\left(\mathbb{R}_{+}\right) \\
H^{-\frac{1}{2}+\varepsilon}\left(\mathbb{R}_{+}\right)
\end{gathered},
$$

in the sense that we relate the operators (5.9) and (5.10) by an operator identity (of the type (6.1) explained below) so that $\mathcal{K}_{--}:=\mathcal{K} \operatorname{diag}\{I-$ $\left.\mathrm{Op}\left(\tau_{-2 a}\right) J, I-\mathrm{Op}\left(\tau_{-2 a}\right) J\right\}$.

Let us note here that due to Theorem 3.1 and having in mind the exhibited limit relations of the potentials, we already know that $\operatorname{Ker} r_{\mathbb{R}_{+}} \mathcal{K}_{--}=\{0\}$.

\subsection{The mixed-N problem}

The boundary value problem $\mathcal{P}_{\text {mixed }-N}$ can equivalently be rewritten [38] in the following form: Find $u_{j} \in H^{1+\varepsilon}\left(\Omega_{j}\right), j=1,2$, such that

$$
\begin{aligned}
& \left(\Delta+k^{2}\right) u_{j}=0 \quad \text { in } \quad \Omega_{j}, \\
& {\left[\partial_{n_{2}} u_{j}\right]_{\mathcal{S}_{j}}^{+}=f_{j} \quad \text { on } \quad \mathcal{S}_{j} \text {, }} \\
& {\left[u_{1}\right]_{\mathcal{C}}^{+}=g_{0}^{+}, \quad\left[\partial_{n_{1}} u_{2}\right]_{\mathcal{C}}^{-}=g_{1}^{-} \quad \text { on } \quad \mathcal{C},}
\end{aligned}
$$


and

$$
\left[u_{1}\right]_{\mathcal{C}^{c}}^{+}-\left[u_{2}\right]_{\mathcal{C}^{c}}^{-}=0, \quad\left[\partial_{n_{1}} u_{1}\right]_{\mathcal{C}^{c}}^{+}-\left[\partial_{n_{1}} u_{2}\right]_{\mathcal{C}^{c}}^{-}=0 \quad \text { on } \quad \mathcal{C}^{c} .
$$

Proposition 5.2. A function $u \in H^{1+\varepsilon}\left(\Omega_{\mathcal{C}}\right)$ is a solution of Problem $\mathcal{P}_{\text {mixed }-N}$ if and only if $u$ can be represented by

$$
u_{1}=2 W_{1}\left(\ell^{e}\left(\ell_{+} g_{0}^{+}+r_{\mathcal{S}} \varphi_{0}\right)\right)-2 V_{2}\left(\ell^{o} f_{1}\right) \text { in } \Omega_{1}
$$

and

$$
\left.u_{2}=2 V_{1}\left(\ell^{e}\left(\ell_{+} g_{1}^{-}-2\left[\partial_{n_{1}} V_{2}\left(\ell^{o} f_{2}\right)\right]_{\mathcal{S}}^{-}\right)+\ell^{e} r_{\mathcal{S}} \varphi_{1}\right)\right)-2 V_{2}\left(\ell^{o} f_{2}\right) \quad \text { in } \Omega_{2},
$$

where $\varphi_{j}$ are suitable elements of the spaces $\widetilde{H}^{\frac{1}{2}+\varepsilon-j}\left(\mathcal{C}^{c}\right), j=0,1$, while $\ell_{+} g_{0}^{+}$ and $\ell_{+} g_{1}^{-}$are above introduced extensions of $g_{0}^{+}$and $g_{1}^{-}$, respectively.

The proof of the last proposition (as well as the ones of the propositions in the next two subsections) are identical to that of Proposition 5.1, and so we shall not write them in a formal way but will simply indicate their main steps. Namely, in the present case, the boundary conditions on $\mathcal{C}^{c}$ lead us to the following equation

$$
r_{\mathcal{C}^{c}} \mathcal{K} \ell^{e} r_{\mathcal{S}} \Upsilon=F^{N}
$$

for the same $\mathcal{K}$ and $\Upsilon$ as in (5.8), and where $F^{N}=\left(F_{1}^{N}, F_{2}^{N}\right)^{\top}$ is a known function with

$F_{0}^{N}:=r_{\mathcal{C}^{c}}\left(2 \mathcal{H}\left(\ell^{e}\left(\ell_{+} g_{1}^{-}-2\left[\partial_{n_{1}} V_{2}\left(\ell^{o} f_{2}\right)\right]\right)\right)-\left[2 V_{2}\left(\ell^{o} f_{2}\right)\right]_{\mathcal{S}}^{-}-\ell_{+} g_{0}^{+}\right) \in H^{\frac{1}{2}+\varepsilon}\left(\mathcal{C}^{c}\right)$ and

$$
F_{1}^{N}:=r_{\mathcal{C}^{c}}\left(\ell_{+} g_{1}^{-}-2 \mathcal{L}\left(\ell^{e}\left(\ell_{+} g_{0}^{+}\right)\right)+2\left[\partial_{n_{1}} V_{2}\left(\ell^{o} f_{1}\right)\right]_{\mathcal{S}}^{+}\right) \in H^{-\frac{1}{2}+\varepsilon}\left(\mathcal{C}^{c}\right) .
$$

Thus we need to investigate the invertibility of the operator

$$
r_{\mathcal{C}^{c}} \mathcal{K} \ell^{e} r_{\mathcal{S}}: \begin{gathered}
\tilde{H}^{\frac{1}{2}+\varepsilon}\left(\mathcal{C}^{c}\right) \\
\widetilde{H}^{-\frac{1}{2}+\varepsilon}\left(\mathcal{C}^{c}\right)
\end{gathered} \longrightarrow \begin{gathered}
H^{\frac{1}{2}+\varepsilon}\left(\mathcal{C}^{c}\right) \\
H^{-\frac{1}{2}+\varepsilon}\left(\mathcal{C}^{c}\right)
\end{gathered} .
$$

As previously, with the help of the operators $J$ and $\mathcal{F}^{-1} \tau_{ \pm a} \cdot \mathcal{F}$, we are able to equivalently transform this second problem into the problem of the invertibility of the operator

$$
r_{\mathbb{R}_{+}} \mathcal{K}_{++}: \begin{gathered}
\widetilde{H}^{\frac{1}{2}+\varepsilon}\left(\mathbb{R}_{+}\right) \\
\widetilde{H}^{-\frac{1}{2}+\varepsilon}\left(\mathbb{R}_{+}\right)
\end{gathered} \longrightarrow \begin{gathered}
H^{\frac{1}{2}+\varepsilon}\left(\mathbb{R}_{+}\right) \\
H^{-\frac{1}{2}+\varepsilon}\left(\mathbb{R}_{+}\right)
\end{gathered}
$$


where $\mathcal{K}_{++}:=\mathcal{K} \operatorname{diag}\left\{I+\mathrm{Op}\left(\tau_{-2 a}\right) J, I+\mathrm{Op}\left(\tau_{-2 a}\right) J\right\}$.

Again, let us note here that due to Theorem 3.1 and having in mind the exhibited limit relations of the potentials, we already know that Ker $r_{\mathbb{R}_{+}} \mathcal{K}_{++}=$ $\{0\}$.

\subsection{The mixed problem}

The boundary value problem $\mathcal{P}_{\text {mixed }}$ can equivalently be rewritten in the following form: Find $u_{j} \in H^{1+\varepsilon}\left(\Omega_{j}\right), j=1,2$, such that

$$
\begin{aligned}
& \left(\Delta+k^{2}\right) u_{j}=0 \quad \text { in } \quad \Omega_{j}, \\
& {\left[\partial_{n_{2}} u_{1}\right]_{\mathcal{S}_{1}}^{+}=f_{1} \quad \text { on } S_{1}, \quad\left[u_{2}\right]_{\mathcal{S}_{2}}^{+}=h_{2} \quad \text { on } \quad \mathcal{S}_{2} \text {, }} \\
& {\left[u_{1}\right]_{\mathcal{C}}^{+}=g_{0}^{+}, \quad\left[\partial_{n_{1}} u_{2}\right]_{\mathcal{C}}^{-}=g_{1}^{-} \quad \text { on } \quad \mathcal{C},}
\end{aligned}
$$

and

$$
\left[u_{1}\right]_{\mathcal{C}^{c}}^{+}-\left[u_{2}\right]_{\mathcal{C}^{c}}^{-}=0, \quad\left[\partial_{n_{1}} u_{1}\right]_{\mathcal{C}^{c}}^{+}-\left[\partial_{n_{1}} u_{2}\right]_{\mathcal{C}^{c}}^{-}=0 \quad \text { on } \quad \mathcal{C}^{c}
$$

where $\mathcal{C}^{c}=\mathcal{S} \backslash \overline{\mathcal{C}}$.

Proposition 5.3. A function $u \in H^{1+\varepsilon}\left(\Omega_{\mathcal{C}}\right)$ is a solution of Problem $\mathcal{P}_{\text {mixed }}$ if and only if $u$ can be represented by

$$
u_{1}=2 W_{1}\left(\ell^{e}\left(\ell_{+} g_{0}^{+}+r_{\mathcal{S}} \varphi_{0}\right)\right)-2 V_{2}\left(\ell^{o} f_{1}\right) \text { in } \Omega_{1}
$$

and

$$
u_{2}=2 V_{1}\left(\ell^{o}\left(\ell_{+} g_{1}^{-}+r_{\mathcal{S}} \varphi_{1}\right)\right)+2 W_{2}\left(\ell^{e} h_{2}\right) \quad \text { in } \Omega_{2},
$$

where $\varphi_{j}$ are suitable elements of the spaces $\widetilde{H}^{\frac{1}{2}+\varepsilon-j}\left(\mathcal{C}^{c}\right), j=0,1$, while $\ell_{+} g_{0}^{+}$ and $\ell_{+} g_{1}^{-}$are above introduced extensions of $g_{0}^{+}$and $g_{1}^{-}$, respectively.

The boundary conditions on $\mathcal{C}^{c}$ lead us to the following equation

$$
r_{\mathcal{C}^{c}} \mathcal{K} \operatorname{diag}\left\{\ell^{e}, \ell^{o}\right\} r_{\mathcal{S}} \Upsilon=F^{M},
$$

where $F^{M}:=\left(F_{0}^{D}, F_{1}^{N}\right)^{\top}$ is a known function directly obtained from (5.15)(5.15) and (4.2)-(4.4). Thus, we need to investigate the invertibility of the operator

$$
r_{\mathcal{C}^{c}} \mathcal{K} \operatorname{diag}\left\{\ell^{e}, \ell^{o}\right\} r_{\mathcal{S}}: \begin{gathered}
\widetilde{H}^{\frac{1}{2}+\varepsilon}\left(\mathcal{C}^{c}\right) \\
\widetilde{H}^{-\frac{1}{2}+\varepsilon}\left(\mathcal{C}^{c}\right)
\end{gathered} \longrightarrow \begin{gathered}
H^{\frac{1}{2}+\varepsilon}\left(\mathcal{C}^{c}\right) \\
H^{-\frac{1}{2}+\varepsilon}\left(\mathcal{C}^{c}\right)
\end{gathered}
$$


As previously, with the help of the operators $J$ and $\mathcal{F}^{-1} \tau_{ \pm a} \cdot \mathcal{F}$, we are able to equivalently transform this second problem into the invertibility of the operator

$$
r_{\mathbb{R}_{+}} \mathcal{K}_{+-}: \begin{gathered}
\widetilde{H}^{\frac{1}{2}+\varepsilon}\left(\mathbb{R}_{+}\right) \\
\widetilde{H}^{-\frac{1}{2}+\varepsilon}\left(\mathbb{R}_{+}\right)
\end{gathered} \longrightarrow \begin{gathered}
H^{\frac{1}{2}+\varepsilon}\left(\mathbb{R}_{+}\right) \\
H^{-\frac{1}{2}+\varepsilon}\left(\mathbb{R}_{+}\right)
\end{gathered}
$$

where $\mathcal{K}_{+-}:=\mathcal{K} \operatorname{diag}\left\{I+\mathrm{Op}\left(\tau_{-2 a}\right) J, I-\mathrm{Op}\left(\tau_{-2 a}\right) J\right\}$.

Again we stress that due to Theorem 3.1 and having in mind the exhibited limit relations of the potentials, we already know that $\operatorname{Ker} r_{\mathbb{R}_{+}} \mathcal{K}_{+-}=\{0\}$.

\subsection{The mixed ${ }^{*}$ problem}

The boundary value problem $\mathcal{P}_{\text {mixed }}$ can equivalently be rewritten in the following form: Find $u_{j} \in H^{1+\varepsilon}\left(\Omega_{j}\right), j=1,2$, such that

$$
\begin{array}{rlll}
\left(\Delta+k^{2}\right) u_{j}=0 & \text { in } \quad \Omega_{j}, \\
{\left[u_{1}\right]_{\mathcal{S}_{1}}^{+}=h_{1} \quad \text { on } S_{1}, \quad\left[\partial_{n_{2}} u_{2}\right]_{\mathcal{S}_{2}}^{+}=f_{2}} & \text { on } \quad \mathcal{S}_{2}, \\
{\left[u_{1}\right]_{\mathcal{C}}^{+}=g_{0}^{+}, \quad\left[\partial_{n_{1}} u_{2}\right]_{\mathcal{C}}^{-}=g_{1}^{-}} & \text {on } \quad \mathcal{C},
\end{array}
$$

and

$$
\left[u_{1}\right]_{\mathcal{C}^{c}}^{+}-\left[u_{2}\right]_{\mathcal{C}^{c}}^{-}=0, \quad\left[\partial_{n_{1}} u_{1}\right]_{\mathcal{C}^{c}}^{+}-\left[\partial_{n_{1}} u_{2}\right]_{\mathcal{C}^{c}}^{-}=0 \quad \text { on } \quad \mathcal{C}^{c} .
$$

Proposition 5.4. A function $u \in H^{1+\varepsilon}\left(\Omega_{\mathcal{C}}\right)$ is a solution of Problem $\mathcal{P}_{\text {mixed }}$ if and only if $u$ can be represented by

$$
u_{1}=2 W_{1}\left(\ell^{o}\left(\ell_{+} g_{0}^{+}-\left[2 W_{2}\left(\ell^{e} h_{1}\right)\right]_{\mathcal{S}}^{+}\right)+\ell^{o} r_{\mathcal{S}} \varphi_{0}\right)+2 W_{2}\left(\ell^{e} h_{1}\right) \quad \text { in } \Omega_{1}
$$

and

$$
\left.u_{2}=2 V_{1}\left(\ell^{e}\left(\ell_{+} g_{1}^{-}-2\left[\partial_{n_{1}} V_{2}\left(\ell^{o} f_{2}\right)\right]_{\mathcal{S}}^{-}\right)+\ell^{e} r_{\mathcal{S}} \varphi_{1}\right)\right)-2 V_{2}\left(\ell^{o} f_{2}\right) \quad \text { in } \Omega_{2},
$$

where $\varphi_{j}$ are suitable elements of the spaces $\widetilde{H}^{\frac{1}{2}+\varepsilon-j}\left(\mathcal{C}^{c}\right), j=0,1$, while $\ell_{+} g_{0}^{+}$ and $\ell_{+} g_{1}^{-}$are above introduced extensions of $g_{0}^{+}$and $g_{1}^{-}$, respectively.

The boundary conditions on $\mathcal{C}^{c}$ lead us to the following equation

$$
r_{\mathcal{C}^{c}} \mathcal{K} \operatorname{diag}\left\{\ell^{o}, \ell^{e}\right\} r_{\mathcal{S}} \Upsilon=F^{M^{*}},
$$


where $F^{M^{*}}:=\left(F_{0}^{N}, F_{1}^{D}\right)^{\top}$ is a known function which is directly constructed from (5.19)-(5.20) and (4.2)-(4.4). Consequently, we need to investigate the invertibility of the operator

$$
r_{\mathcal{C}^{c}} \mathcal{K} \operatorname{diag}\left\{\ell^{o}, \ell^{e}\right\} r_{\mathcal{S}}: \begin{gathered}
\widetilde{H}^{\frac{1}{2}+\varepsilon}\left(\mathcal{C}^{c}\right) \\
\widetilde{H}^{-\frac{1}{2}+\varepsilon}\left(\mathcal{C}^{c}\right)
\end{gathered} \longrightarrow \begin{gathered}
H^{\frac{1}{2}+\varepsilon}\left(\mathcal{C}^{c}\right) \\
H^{-\frac{1}{2}+\varepsilon}\left(\mathcal{C}^{c}\right)
\end{gathered} .
$$

As previously, with the help of the operators $J$ and $\mathcal{F}^{-1} \tau_{ \pm a} \cdot \mathcal{F}$, we are able to equivalently transform this second problem into the question of the invertibility of the operator

$$
r_{\mathbb{R}_{+}} \mathcal{K}_{-+}: \begin{gathered}
\widetilde{H}^{\frac{1}{2}+\varepsilon}\left(\mathbb{R}_{+}\right) \\
\widetilde{H}^{-\frac{1}{2}+\varepsilon}\left(\mathbb{R}_{+}\right)
\end{gathered} \longrightarrow \begin{gathered}
H^{\frac{1}{2}+\varepsilon}\left(\mathbb{R}_{+}\right) \\
H^{-\frac{1}{2}+\varepsilon}\left(\mathbb{R}_{+}\right)
\end{gathered},
$$

where $\mathcal{K}_{-+}:=\mathcal{K} \operatorname{diag}\left\{I-\mathrm{Op}\left(\tau_{-2 a}\right) J, I+\mathrm{Op}\left(\tau_{-2 a}\right) J\right\}$.

Again, let us note here that due to Theorem 3.1 and having in mind the exhibited limit relations of the potentials, we already know that $\operatorname{Ker} r_{\mathbb{R}_{+}} \mathcal{K}_{-+}=$ $\{0\}$.

\section{Operator relations and Fredholm theory}

Now, we will exhibit operator relations for the operators which appeared in the last section, and which will help us in obtaining their Fredholm properties at the end of the present section.

In order to make this happen, we start by recalling that two bounded linear operators $T: X_{1} \rightarrow X_{2}$ and $S: Y_{1} \rightarrow Y_{2}$, acting between Banach spaces, are said to be (toplinear) equivalent after extension if there are Banach spaces $Z_{1}$ and $Z_{2}$ and invertible bounded linear operators $E$ and $F$ such that

$$
\left[\begin{array}{cc}
T & 0 \\
0 & I_{Z_{1}}
\end{array}\right]=E\left[\begin{array}{cc}
S & 0 \\
0 & I_{Z_{2}}
\end{array}\right] F
$$

where $I_{Z_{1}}$ and $I_{Z_{2}}$ represent the identity operators in $Z_{1}$ and $Z_{2}$, respectively. In particular, in case we will simply have $T=E S F$ for some boundedly invertible operators $E$ and $F$, we will say that $T$ and $S$ are equivalent operators. In such a case, we will write $T \sim S$. These operator relations between two 
operators $T$ and $S$, if obtained, allow several consequences on the properties of these two operators. Namely, $T$ and $S$ will have the same Fredholm regularity properties (i.e., the properties that directly depend on the kernel and on the image of the operator). Cf. [2, 16] for a detailed description about such operator relations and their properties.

Let us define

$$
\Lambda_{ \pm}^{s}(\xi):=(\xi \pm i)^{s}=\left(1+\xi^{2}\right)^{\frac{s}{2}} \exp \{s i \arg (\xi \pm i)\},
$$

with a branch chosen in such a way that $\arg (\xi \pm i) \rightarrow 0$ as $\xi \rightarrow+\infty$, i.e., with a cut parallel to the negative real axis (see Example 1.7 in [21] for additional information about the properties of these functions). In addition, we will also use the notation

$$
\zeta(\xi):=\frac{\Lambda_{-}(\xi)}{\Lambda_{+}(\xi)}=\frac{\xi-i}{\xi+i}, \quad \xi \in \mathbb{R} .
$$

Lemma 6.1. [21, §4] Let $s, r \in \mathbb{R}$, and consider the operators

$$
\begin{aligned}
\Lambda_{+}^{s}(D) & =(D+i)^{s} \\
\Lambda_{-}^{s}(D) & =r_{\mathbb{R}_{+}}(D-i)^{s} \ell^{(r)},
\end{aligned}
$$

where $(D \pm i)^{ \pm s}=\mathcal{F}^{-1}(\xi \pm i)^{ \pm s} \cdot \mathcal{F}$, and $\ell^{(r)}: H^{r}\left(\mathbb{R}_{+}\right) \rightarrow H^{r}(\mathbb{R})$ is any bounded extension operator in these spaces (which particular choice does not change the definition of $\left.\Lambda_{-}^{s}(D)\right)$.

These operators arrange isomorphisms in the following space settings

$$
\begin{aligned}
& \Lambda_{+}^{s}(D): \quad \widetilde{H}^{r}\left(\mathbb{R}_{+}\right) \rightarrow \widetilde{H}^{r-s}\left(\mathbb{R}_{+}\right), \\
& \Lambda_{-}^{s}(D): H^{r}\left(\mathbb{R}_{+}\right) \rightarrow H^{r-s}\left(\mathbb{R}_{+}\right)
\end{aligned}
$$

for any $s, r \in \mathbb{R}$.

Bearing in mind the purpose of this section, let $A_{i j}=\operatorname{Op}\left(a_{i j}\right)=\mathcal{F}^{-1} a_{i j} \cdot \mathcal{F}$ and $B_{i j}=\mathrm{Op}\left(b_{i j}\right)$ be pseudodifferential operators of order $\mu_{i j} \in \mathbb{R}$; thus, $\langle\cdot\rangle^{-\mu_{i j}} a_{i j},\langle\cdot\rangle^{-\mu_{i j}} b_{i j} \in L^{\infty}(\mathbb{R})$, where $\langle\xi\rangle:=\left(1+\xi^{2}\right)^{\frac{1}{2}}$ and $i, j=1,2$. Since the operators $r_{\mathbb{R}_{+}}\left(A_{i j}+B_{i j} J\right)$ arrange continuous maps

$$
r_{\mathbb{R}_{+}}\left(A_{i j}+B_{i j} J\right): \widetilde{H}^{s}\left(\mathbb{R}_{+}\right) \rightarrow H^{s-\mu_{i j}}\left(\mathbb{R}_{+}\right)
$$


for all $s \in \mathbb{R}$, then the $2 \times 2$ matrix operator

$$
A+B J=\left(\begin{array}{cc}
A_{11}+B_{11} J & A_{12}+B_{12} J \\
A_{21}+B_{21} J & A_{22}+B_{22} J
\end{array}\right), \quad A=\left(A_{i j}\right)_{i, j=1,2}, \quad B=\left(B_{i j}\right)_{i, j=1,2}
$$

arranges a continuous map

$$
r_{\mathbb{R}_{+}}(A+B J): \begin{gathered}
\widetilde{H}^{\frac{1}{2}+\varepsilon}\left(\mathbb{R}_{+}\right) \\
\widetilde{H}^{-\frac{1}{2}+\varepsilon}\left(\mathbb{R}_{+}\right)
\end{gathered} \rightarrow \begin{gathered}
H^{\frac{1}{2}+\varepsilon}\left(\mathbb{R}_{+}\right) \\
H^{-\frac{1}{2}+\varepsilon}\left(\mathbb{R}_{+}\right)
\end{gathered}
$$

where $A_{11}=A_{22}=I, A_{12}=2 \mathcal{H}, A_{21}=2 \mathcal{L}$, and $B_{i j}=A_{i j} \mathrm{Op}\left(\tau_{-2 a}\right)$, for $i, j=1,2$.

Recall that the complete symbol of the pseudodifferential operators $\mathcal{H}$ and $\mathcal{L}$ are (cf. $[7,10])$ :

$$
\sigma(\mathcal{H})(\xi)=-\frac{i}{2 w(\xi)} \quad \text { and } \quad \sigma(\mathcal{L})(\xi)=-\frac{i w(\xi)}{2}
$$

where $w=w(\xi):=\left(\varrho^{2}+\rho^{2}\right)^{\frac{1}{4}}\left(\cos \frac{\alpha}{2}+i \sin \frac{\alpha}{2}\right)$, with

$$
\begin{aligned}
& \varrho=\varrho(\xi):=(\Re \mathrm{e} k)^{2}-(\Im \mathrm{m} k)^{2}-\xi^{2}, \\
& \rho:=2(\Re \mathrm{e} k)(\Im \mathrm{m} k)
\end{aligned}
$$

and

$$
\alpha:=\left\{\begin{array}{lll}
\arctan \frac{\rho}{|\varrho|} & \text { if } \quad \varrho>0, \rho>0 \\
\frac{\pi}{2} & \text { if } \quad \varrho=0, \rho>0 \\
\pi-\arctan \frac{\rho}{|\varrho|} & \text { if } \quad \varrho<0, \rho>0 \\
\pi & \text { if } \quad \rho=0 \\
2 \pi-\arctan \frac{|\rho|}{|\varrho|} & \text { if } \quad \varrho>0, \rho<0 \\
\frac{3 \pi}{2} & \text { if } \quad \varrho=0, \rho<0 \\
\pi+\arctan \frac{|\rho|}{|\varrho|} & \text { if } \quad \varrho<0, \rho<0
\end{array} .\right.
$$

Lemma 6.1 allows us to construct an equivalence relation between $r_{\mathbb{R}_{+}}(A+$ $B J)$ and

$$
r_{\mathbb{R}_{+}}(\mathcal{A}+\mathcal{B} J):\left[L_{2}\left(\mathbb{R}_{+}\right)\right]^{2} \rightarrow\left[L_{2}\left(\mathbb{R}_{+}\right)\right]^{2},
$$

which is explicitly given by the following identity

$$
r_{\mathbb{R}_{+}}(\mathcal{A}+\mathcal{B} J):=\operatorname{diag}\left\{\Lambda_{-}^{\frac{1}{2}+\varepsilon}, \Lambda_{-}^{-\frac{1}{2}+\varepsilon}\right\} r_{\mathbb{R}_{+}}(A+B J) \operatorname{diag}\left\{\Lambda_{+}^{-\frac{1}{2}-\varepsilon}, \Lambda_{+}^{\frac{1}{2}-\varepsilon}\right\}(6.6)
$$


where $\mathcal{A}:=\left(\mathcal{A}_{i j}\right)_{i, j=1,2}, \mathcal{B}:=\left(\mathcal{B}_{i j}\right)_{i, j=1,2}$, with

$$
\mathcal{A}_{i j}:=(D-i)^{r_{i}} A_{i j}(D+i)^{-r_{j}}, \quad \mathcal{B}_{i j}:=(D-i)^{r_{i}} B_{i j} J(D+i)^{-r_{j}} J,
$$

for $r_{1}:=\frac{1}{2}+\varepsilon, r_{2}:=-\frac{1}{2}+\varepsilon$. Due to the fact that $\Lambda_{-}^{s-\mu}: H^{s-\mu}\left(\mathbb{R}_{+}\right) \rightarrow L_{2}\left(\mathbb{R}_{+}\right)$ and $\Lambda_{+}^{-s}: L_{2}\left(\mathbb{R}_{+}\right) \rightarrow \widetilde{H}^{s}\left(\mathbb{R}_{+}\right)$are invertible operators (cf. Lemma 6.1), the identity (6.6) shows that

$$
r_{\mathbb{R}_{+}}(A+B J) \sim r_{\mathbb{R}_{+}}(\mathcal{A}+\mathcal{B} J) .
$$

Note that

$$
\Lambda_{+}^{s}(-\xi)=\Lambda_{-}^{s}(\xi) e^{s \pi i}, \quad \Lambda_{-}^{s}(-\xi)=\Lambda_{+}^{s}(\xi) e^{-s \pi i}
$$

which in particular allow us to describe the operators $\mathcal{A}_{i j}$ and $\mathcal{B}_{i j}$ and their symbols in the following way

$$
\begin{aligned}
\mathcal{A}_{i j}=\operatorname{Op}\left(\tilde{a}_{i j}\right), & & \tilde{a}_{i j}(\xi)=\Lambda_{-}^{r_{i}}(\xi) a_{i j}(\xi) \Lambda_{+}^{-r_{j}}(\xi), \\
\mathcal{B}_{i j}=\operatorname{Op}\left(\tilde{b}_{i j}\right), & & \tilde{b}_{i j}(\xi)=\Lambda_{-}^{r_{i}}(\xi) b_{i j}(\xi) \Lambda_{+}^{-r_{j}}(-\xi)=\Lambda_{-}^{r_{i}-r_{j}}(\xi) b_{i j}(\xi) e^{-r_{j} \pi i} .
\end{aligned}
$$

In particular, we have $\sigma(\mathcal{A})(\xi)=\left(\tilde{a}_{i j}(\xi)\right)_{i, j=1,2}$ with

$$
\begin{aligned}
\tilde{a}_{11}(\xi) & =\zeta^{\frac{1}{2}+\varepsilon}(\xi), & & \tilde{a}_{12}(\xi)=2 \zeta^{\varepsilon}(\xi)\langle\xi\rangle \sigma(\mathcal{H})(\xi), \\
\tilde{a}_{21}(\xi) & =2 \zeta^{\varepsilon}(\xi)\langle\xi\rangle^{-1} \sigma(\mathcal{L})(\xi), & & \tilde{a}_{22}(\xi)=\zeta^{-\frac{1}{2}+\varepsilon}(\xi),
\end{aligned}
$$

and $\sigma(\mathcal{B})(\xi)=\left(\tilde{b}_{i j}(\xi)\right)_{i, j=1,2}$, where

$$
\begin{aligned}
& \tilde{b}_{11}(\xi)=-i \tau_{-2 a}(\xi) e^{-\varepsilon \pi i}, \quad \tilde{b}_{12}(\xi)=2 i(\xi-i) \tau_{-2 a}(\xi) \sigma(\mathcal{H})(\xi) e^{-\varepsilon \pi i} \\
& \tilde{b}_{21}(\xi)=-2 i(\xi-i)^{-1} \tau_{-2 a}(\xi) \sigma(\mathcal{L})(\xi) e^{-\varepsilon \pi i}, \quad \tilde{b}_{22}(\xi)=i \tau_{-2 a}(\xi) e^{-\varepsilon \pi i}
\end{aligned}
$$

Thus

$$
r_{\mathbb{R}_{+}} \mathcal{K}_{++} \sim r_{\mathbb{R}_{+}}(\mathcal{A}+\mathcal{B} J) \text { and } \quad r_{\mathbb{R}_{+}} \mathcal{K}_{--} \sim r_{\mathbb{R}_{+}}(\mathcal{A}-\mathcal{B} J) .
$$

Similarly, we obtain

$$
r_{\mathbb{R}_{+}} \mathcal{K}_{+-} \sim r_{\mathbb{R}_{+}}\left(\mathcal{A}+\mathcal{B}^{\prime} J\right) \text { and } \quad r_{\mathbb{R}_{+}} \mathcal{K}_{-+} \sim r_{\mathbb{R}_{+}}\left(\mathcal{A}-\mathcal{B}^{\prime} J\right)
$$

where $\mathcal{B}^{\prime}:=\operatorname{diag}\{1,-1\} \mathcal{B}$. 
From now on we provide detailed arguments for the operators given in (6.9), while the operators in (6.10) can be treated analogously with obvious changes in the corresponding places.

Further, let us consider a pseudodifferential operator $\operatorname{Op}(\Xi)$ with $4 \times 4$ matrix symbol $\Xi(\xi)$ partitioned into four $2 \times 2$ blocks $\alpha_{i j}, i, j=1,2$ :

$$
\Xi(\xi)=\left(\begin{array}{ll}
\alpha_{11}(\xi) & \alpha_{12}(\xi) \\
\alpha_{21}(\xi) & \alpha_{22}(\xi)
\end{array}\right)
$$

with

$$
\begin{aligned}
\alpha_{11}(\xi) & =\sigma(\mathcal{A})(\xi)-\sigma(\mathcal{B})(\xi)[\sigma(\mathcal{A})(-\xi)]^{-1} \sigma(\mathcal{B})(-\xi), \\
\alpha_{12}(\xi) & =-\sigma(\mathcal{B})(\xi)[\sigma(\mathcal{A})(-\xi)]^{-1} \\
\alpha_{21}(\xi) & =[\sigma(\mathcal{A})(-\xi)]^{-1} \sigma(\mathcal{B})(-\xi) \\
\alpha_{22}(\xi) & =(\sigma(\mathcal{A})(-\xi))^{-1}
\end{aligned}
$$

The direct calculation shows that $\alpha_{11}$ is the null matrix, i.e., $\alpha_{11}(\xi) \equiv 0$, while

$$
\begin{aligned}
& \alpha_{12}(\xi)=\left(\begin{array}{cc}
-i \tau_{-2 a}(\xi) e^{\varepsilon \pi i} \zeta^{\frac{1}{2}+\varepsilon}(\xi) & 0 \\
0 & i \tau_{-2 a}(\xi) e^{\varepsilon \pi i} \zeta^{-\frac{1}{2}+\varepsilon}(\xi)
\end{array}\right), \\
& \alpha_{21}(\xi)=\left(\begin{array}{cc}
i \tau_{2 a}(\xi) e^{\varepsilon \pi i} \zeta^{\frac{1}{2}+\varepsilon}(\xi) & 0 \\
0 & -i \tau_{2 a}(\xi) e^{\varepsilon \pi i} \zeta^{-\frac{1}{2}+\varepsilon}(\xi)
\end{array}\right), \\
& \alpha_{22}(\xi)=\left(\begin{array}{cc}
-\frac{1}{2} e^{2 \varepsilon \pi i} \zeta^{\frac{1}{2}+\varepsilon}(\xi) & -e^{2 \varepsilon \pi i}\langle\xi\rangle \sigma(\mathcal{H}) \zeta^{\varepsilon}(\xi) \\
-e^{2 \varepsilon \pi i}\langle\xi\rangle^{-1} \sigma(\mathcal{L}) \zeta^{\varepsilon}(\xi) & -\frac{1}{2} e^{2 \varepsilon \pi i} \zeta^{-\frac{1}{2}+\varepsilon}(\xi)
\end{array}\right) .
\end{aligned}
$$

Under the above conditions it is straightforward to conclude that

$$
r_{\mathbb{R}_{+}} \mathrm{Op}(\Xi):\left[L_{2}\left(\mathbb{R}_{+}\right)\right]^{4} \rightarrow\left[L_{2}\left(\mathbb{R}_{+}\right)\right]^{4}
$$

is a continuous operator. Moreover, it is easy to see that the determinant of the symbol of this operator is always nonzero, for all $\xi \in \mathbb{R}$.

The importance of the operator $r_{\mathbb{R}_{+}} \mathrm{Op}(\Xi)$ is clarified by the next result.

Theorem 6.2. (i) The operators

$$
r_{\mathbb{R}_{+}} \mathcal{A} \pm r_{\mathbb{R}_{+}} \mathcal{B} J:\left[L_{2}\left(\mathbb{R}_{+}\right)\right]^{2} \rightarrow\left[L_{2}\left(\mathbb{R}_{+}\right)\right]^{2}
$$

(defined in (6.5)-(6.7)) are both invertible if and only if the operator $r_{\mathbb{R}_{+}} \mathrm{Op}(\Xi)$ (given in (6.14)) is invertible. 
(ii) The operators $r_{\mathbb{R}_{+}} \mathcal{A}+r_{\mathbb{R}_{+}} \mathcal{B} J$ and $r_{\mathbb{R}_{+}} \mathcal{A}-r_{\mathbb{R}_{+}} \mathcal{B} J$ have both the Fredholm property if and only if $r_{\mathbb{R}_{+}} \mathrm{Op}(\Xi)$ has the Fredholm property. In addition, when in the presence of the Fredholm property for these three operators, their Fredholm indices satisfy the identity

$$
\text { Ind }\left(r_{\mathbb{R}_{+}} \mathcal{A}+r_{\mathbb{R}_{+}} \mathcal{B} J\right)+\operatorname{Ind}\left(r_{\mathbb{R}_{+}} \mathcal{A}-r_{\mathbb{R}_{+}} \mathcal{B} J\right)=\operatorname{Ind} r_{\mathbb{R}_{+}} \mathrm{Op}(\Xi) \text {. }
$$

In fact, this theorem is a consequence of a stronger fact which basically states that $r_{\mathbb{R}_{+}} \mathrm{Op}(\Xi)$ is (toplinear) equivalent after extension with a diagonal block matrix operator whose diagonal entries are the operators $r_{\mathbb{R}_{+}} \mathcal{A}+r_{\mathbb{R}_{+}} \mathcal{B} J$ and $r_{\mathbb{R}_{+}} \mathcal{A}-r_{\mathbb{R}_{+}} \mathcal{B} J$. Moreover, it is interesting to clarify that all the necessary operators to identify such (toplinear) equivalence after extension relation can be built in an explicit way (see $[13,14,15,16])$.

\section{Associated Wiener-Hopf operators: their Fredholm properties and factorization}

In this section we would like to investigate the Wiener-Hopf operator obtained in the previous section

$$
r_{\mathbb{R}_{+}} \mathrm{Op}(\Xi):\left[L_{2}\left(\mathbb{R}_{+}\right)\right]^{4} \rightarrow\left[L_{2}\left(\mathbb{R}_{+}\right)\right]^{4} .
$$

We have that $\Xi$ belongs to the very general $C^{*}$-algebra of the semi-almost periodic four by four matrix functions on the real line $\left([S A P(\mathbb{R})]^{4 \times 4}\right)$; see $[42]$. We recall that $[S A P(\mathbb{R})]^{4 \times 4}$ is the smallest closed subalgebra of $\left[L^{\infty}(\mathbb{R})\right]^{4 \times 4}$ that contains the (classical) algebra of (two by two) almost periodic elements $\left([A P]^{4 \times 4}\right)$ and the (four by four) continuous matrices with possible jumps at infinity.

Due to a known characterization of the structure of $[S A P(\mathbb{R})]^{4 \times 4}$ (see $[3,4,42])$, we can choose a continuous function on the real line, say $\gamma$, such that $\gamma(-\infty)=0, \gamma(+\infty)=1$ and

$$
\Xi=(1-\gamma) \Xi_{l}+\gamma \Xi_{r}+\Xi_{0}
$$

where $\Xi_{0}$ is a continuous four by four matrix function with zero limit at infinity, and $\Xi_{l}$ and $\Xi_{r}$ are matrices with almost periodic elements, uniquely determined by $\Xi$, and that in our case have the following form (due to the 
behavior of $\Xi$ at $\pm \infty)$ :

$$
\begin{aligned}
& \Xi_{l}=\left(\begin{array}{cccc}
0 & 0 & i \tau_{-2 a} e^{-\varepsilon \pi i} & 0 \\
0 & 0 & 0 & -i \tau_{-2 a} e^{-\varepsilon \pi i} \\
-i \tau_{2 a} e^{-\varepsilon \pi i} & 0 & \frac{1}{2} & \frac{1}{2} \\
0 & i \tau_{2 a} e^{-\varepsilon \pi i} & -\frac{1}{2} & \frac{1}{2}
\end{array}\right), \\
& \Xi_{r}=\left(\begin{array}{cccc}
0 & 0 & -i \tau_{-2 a} e^{\varepsilon \pi i} & 0 \\
0 & 0 & 0 & i \tau_{-2 a} e^{\varepsilon \pi i} \\
i \tau_{2 a} e^{\varepsilon \pi i} & 0 & -\frac{1}{2} e^{2 \varepsilon \pi i} & \frac{1}{2} e^{2 \varepsilon \pi i} \\
0 & -i \tau_{2 a} e^{\varepsilon \pi i} & -\frac{1}{2} e^{2 \varepsilon \pi i} & -\frac{1}{2} e^{2 \varepsilon \pi i}
\end{array}\right) .
\end{aligned}
$$

Here, it is worth noting that we had in consideration that $\omega(\xi) \rightarrow i|\xi|$ as $\xi \rightarrow \pm \infty$ (cf. (6.3)-(6.4)), and

$$
\zeta^{\nu}(\xi) \rightarrow 1 \text { as } \xi \rightarrow \infty
$$

and

$$
\zeta^{\nu}(\xi) \rightarrow e^{-2 \pi \nu i} \quad \text { as } \quad \xi \rightarrow-\infty .
$$

For a given unital Banach algebra $\mathcal{M}$, by $\mathcal{G M}$ we will denote the group of all invertible elements of $\mathcal{M}$.

Definition 7.1 (See, e.g., [23] or $\S 6.3$ in [5]). An invertible almost periodic matrix function $\Phi \in \mathcal{G}[A P]^{4 \times 4}$ admits a canonical right AP-factorization if

$$
\Phi=\Phi^{-} \Phi^{+}
$$

where $\Phi^{ \pm} \in \mathcal{G}\left[A P^{ \pm}\right]^{4 \times 4}$, with $A P^{ \pm}$denoting the intersection of $A P$ with the non-tangential limits of functions in $H^{\infty}\left(\mathbb{C}_{ \pm}\right)$(the set of all bounded and analytic functions in $\mathbb{C}_{ \pm}$).

Proposition 7.2. (cf., e.g., [5, Proposition 2.22]) Let $A \subset(0, \infty)$ be an unbounded set and let

$$
\left\{I_{\alpha}\right\}_{\alpha \in A}=\left\{\left(x_{\alpha}, y_{\alpha}\right)\right\}_{\alpha \in A}
$$


be a family of intervals $I_{\alpha} \subset \mathbb{R}$ such that $\left|I_{\alpha}\right|=y_{\alpha}-x_{\alpha} \rightarrow \infty$ as $\alpha \rightarrow \infty$. If $\varphi \in A P$, then the limit

$$
M(\varphi):=\lim _{\alpha \rightarrow \infty} \frac{1}{\left|I_{\alpha}\right|} \int_{I_{\alpha}} \varphi(x) d x
$$

exists, is finite, and is independent of the particular choice of the family $\left\{I_{\alpha}\right\}$.

Definition 7.3. (i) For any $\varphi \in A P$, the number that has just been introduced in Proposition 7.2, $M(\varphi)$, is called the Bohr mean value (or simply the mean value) of $\varphi$. In the matrix case the Bohr mean value is defined entry-wise.

(ii) For a matrix function $\Phi \in \mathcal{G}[A P]^{4 \times 4}$ admitting a canonical right $A P$ factorization (7.2), we may define the new matrix

$$
\mathbf{d}(\Phi):=M\left(\Phi^{-}\right) M\left(\Phi^{+}\right),
$$

which is known as the geometric mean of $\Phi$.

It is worth mentioning that (7.3) is independent of the particular choice of the (canonical) right $A P$ factorization of $\Phi$, and that this definition is consistent with the corresponding one for the scalar case (which can be defined in a somehow more global way).

Theorem 7.4. For $0 \leq \varepsilon<1 / 4$, the operator $r_{\mathbb{R}_{+}} \mathrm{Op}(\Xi):\left[L_{2}\left(\mathbb{R}_{+}\right)\right]^{4} \rightarrow$ $\left[L_{2}\left(\mathbb{R}_{+}\right)\right]^{4}$ is a Fredholm operator with zero Fredholm index.

Proof. The matrices $\Xi_{l}$ and $\Xi_{r}$ admit the following right canonical $A P$ factorizations:

$$
\Xi_{l}=\left(\Xi_{l}\right)_{l}\left(\Xi_{l}\right)_{r}, \quad \text { and } \quad \Xi_{r}=\left(\Xi_{r}\right)_{l}\left(\Xi_{r}\right)_{r},
$$

where

$$
\begin{aligned}
& \left(\Xi_{l}\right)_{l}=\left(\begin{array}{cccc}
e^{-2 \varepsilon \pi i} & e^{-2 \varepsilon \pi i} & i \tau_{-2 a} e^{-\varepsilon \pi i} & 0 \\
0 & e^{-2 \varepsilon \pi i} & -i \tau_{-2 a} e^{-\varepsilon \pi i} & -2 i \tau_{-2 a} e^{-\varepsilon \pi i} \\
0 & 0 & 1 & 1 \\
0 & 0 & 0 & 1
\end{array}\right), \\
& \left(\Xi_{l}\right)_{r}=\left(\begin{array}{cccc}
-2 & 0 & 0 & 0 \\
1 & -1 & 0 & 0 \\
-i \tau_{2 a} e^{-\varepsilon \pi i} & -i \tau_{2 a} e^{-\varepsilon \pi i} & 1 & 0 \\
0 & i \tau_{2 a} e^{-\varepsilon \pi i} & -\frac{1}{2} & \frac{1}{2}
\end{array}\right)
\end{aligned}
$$




$$
\begin{aligned}
\left(\Xi_{r}\right)_{l}= & \left(\begin{array}{cccc}
1 & 1 & i \tau_{-2 a} e^{-\varepsilon \pi i} & 0 \\
0 & 1 & -i \tau_{-2 a} e^{-\varepsilon \pi i} & 2 i \tau_{-2 a} e^{-\varepsilon \pi i} \\
0 & 0 & -1 & 1 \\
0 & 0 & 0 & -1
\end{array}\right), \\
\left(\Xi_{r}\right)_{r}= & \left(\begin{array}{cccc}
-2 & 0 & 0 & 0 \\
1 & 1 & 0 & 0 \\
-i \tau_{2 a} e^{\varepsilon \pi i} & i \tau_{2 a} e^{\varepsilon \pi i} & e^{2 \varepsilon \pi i} & 0 \\
0 & i \tau_{2 a} e^{\varepsilon \pi i} & \frac{1}{2} e^{2 \varepsilon \pi i} & \frac{1}{2} e^{2 \varepsilon \pi i}
\end{array}\right)
\end{aligned}
$$

(in which the necessary factor properties are evident; cf. Definition 7.1).

Having built the factorizations (7.4), we can now apply Theorem 3.2 in [23] or Theorem 10.11 in [5] in view of proving the Fredholm property for $r_{\mathbb{R}_{+}} \mathrm{Op}(\Xi)$. Indeed, within our case of $\Xi \in \mathcal{G}[S A P(\mathbb{R})]^{4 \times 4}$ and whose local representatives at infinity admit canonical right $A P$-factorizations (7.4), applying that theorems we have that $r_{\mathbb{R}_{+}} \mathrm{Op}(\Xi)$ is a Fredholm operator if and only if

$$
\operatorname{sp}\left[\mathbf{d}^{-1}\left(\Xi_{r}\right) \mathbf{d}\left(\Xi_{l}\right)\right] \cap(-\infty, 0]=\emptyset,
$$

where $\operatorname{sp}\left[\mathbf{d}^{-1}\left(\Xi_{r}\right) \mathbf{d}\left(\Xi_{l}\right)\right]$ stands for the set of eigenvalues of the matrix

$$
\mathbf{d}^{-1}\left(\Xi_{r}\right) \mathbf{d}\left(\Xi_{l}\right):=\left[\mathbf{d}\left(\Xi_{r}\right)\right]^{-1} \mathbf{d}\left(\Xi_{l}\right) .
$$

Noticing that directly from the definition of the Bohr mean value we have 
$M( \pm 1 / 2)= \pm 1 / 2, M( \pm 1)= \pm 1, M(-2)=-2$, and $M\left(\tau_{ \pm 2 a}\right)=0$, it follows

$$
\begin{aligned}
\mathbf{d}\left(\Xi_{l}\right) & =M\left[\left(\Xi_{l}\right)_{l}\right] M\left[\left(\Xi_{l}\right)_{r}\right] \\
& =\left(\begin{array}{cccc}
e^{-2 \varepsilon \pi i} & e^{-2 \varepsilon \pi i} & 0 & 0 \\
0 & e^{-2 \varepsilon \pi i} & 0 & 0 \\
0 & 0 & 1 & 1 \\
0 & 0 & 0 & 1
\end{array}\right)\left(\begin{array}{cccc}
-2 & 0 & 0 & 0 \\
1 & -1 & 0 & 0 \\
0 & 0 & 1 & 0 \\
0 & 0 & -\frac{1}{2} & \frac{1}{2}
\end{array}\right) \\
& =\left(\begin{array}{cccc}
-e^{-2 \varepsilon \pi i} & -e^{-2 \varepsilon \pi i} & 0 & 0 \\
e^{-2 \varepsilon \pi i} & -e^{-2 \varepsilon \pi i} & 0 & 0 \\
0 & 0 & \frac{1}{2} & \frac{1}{2} \\
0 & 0 & -\frac{1}{2} & \frac{1}{2}
\end{array}\right),
\end{aligned}
$$

$$
\mathbf{d}\left(\Xi_{r}\right)=M\left[\left(\Xi_{r}\right)_{l}\right] M\left[\left(\Xi_{r}\right)_{r}\right]=\left(\begin{array}{cccc}
-1 & 1 & 0 & 0 \\
1 & 1 & 0 & 0 \\
0 & 0 & -\frac{1}{2} e^{2 \varepsilon \pi i} & \frac{1}{2} e^{2 \varepsilon \pi i} \\
0 & 0 & -\frac{1}{2} e^{2 \varepsilon \pi i} & -\frac{1}{2} e^{2 \varepsilon \pi i}
\end{array}\right) .
$$

As a consequence,

$$
\mathbf{d}^{-1}\left(\Xi_{r}\right) \mathbf{d}\left(\Xi_{l}\right)=\left(\begin{array}{cccc}
e^{-2 \varepsilon \pi i} & 0 & 0 & 0 \\
0 & -e^{-2 \varepsilon \pi i} & 0 & 0 \\
0 & 0 & 0 & -e^{-2 \varepsilon \pi i} \\
0 & 0 & e^{-2 \varepsilon \pi i} & 0
\end{array}\right)
$$

and

$$
\operatorname{sp}\left[\mathbf{d}^{-1}\left(\Xi_{r}\right) \mathbf{d}\left(\Xi_{l}\right)\right] \cap(-\infty, 0]=\left\{ \pm e^{-2 \varepsilon \pi i}, \pm i e^{-2 \varepsilon \pi i}\right\} \cap(-\infty, 0]=\emptyset,(7.7)
$$

which allows us to conclude that $r_{\mathbb{R}_{+}} \mathrm{Op}(\Xi):\left[L_{2}\left(\mathbb{R}_{+}\right)\right]^{4} \rightarrow\left[L_{2}\left(\mathbb{R}_{+}\right)\right]^{4}$ is a Fredholm operator (for the case in consideration of $0 \leq \varepsilon<1 / 4$ ).

The zero Fredholm index is obtained from the formula (cf. Theorem 10.21 in $[5])$

$$
\text { Ind } r_{\mathbb{R}_{+}} \mathrm{Op}(\Xi)=- \text { ind }[\operatorname{det} \Xi]-\sum_{j=1}^{4}\left(\frac{1}{2}-\left\{\frac{1}{2}-\frac{1}{2 \pi} \arg \xi_{j}\right\}\right),
$$


where ind $[\operatorname{det} \Xi]$ denotes the Cauchy index of the determinant of $\Xi$, the numbers $\xi_{j} \in \mathbb{C} \backslash(-\infty, 0], j=1, \ldots, 4$ are the eigenvalues of the matrix $\mathbf{d}^{-1}\left(\Xi_{r}\right) \mathbf{d}\left(\Xi_{l}\right)$ and $\{\cdot\}$ stands for the fractional part of a real number.

Remark 7.5. From the proof of the last result, and in particular from (7.7), we realize that if we would allow the case $\varepsilon=1 / 4$ then our operators would not have the Fredholm property (and therefore would not be invertible operators).

Corollary 7.6. Let $0 \leq \varepsilon<\frac{1}{4}$. The Wiener-Hopf plus and minus Hankel operators (5.10), (5.14), (5.18), and (5.22) (which characterize our four problems) are invertible operators.

Proof. Bearing in mind the equivalence relations (6.9), we have:

$$
\begin{aligned}
\operatorname{dim} \operatorname{CoKer} r_{\mathbb{R}_{+}} \mathcal{K}_{++} & =\operatorname{dim} \operatorname{CoKer} r_{\mathbb{R}_{+}}(\mathcal{A}+\mathcal{B} J) \\
\operatorname{dim} \operatorname{Ker} r_{\mathbb{R}_{+}} \mathcal{K}_{++} & =\operatorname{dim} \operatorname{Ker} r_{\mathbb{R}_{+}}(\mathcal{A}+\mathcal{B} J) .
\end{aligned}
$$

and

$$
\begin{aligned}
\operatorname{dim} \operatorname{CoKer} r_{\mathbb{R}_{+}} \mathcal{K}_{--} & =\operatorname{dim} \operatorname{CoKer} r_{\mathbb{R}_{+}}(\mathcal{A}-\mathcal{B} J) \\
\operatorname{dim} \operatorname{Ker} r_{\mathbb{R}_{+}} \mathcal{K}_{--} & =\operatorname{dim} \operatorname{Ker} r_{\mathbb{R}_{+}}(\mathcal{A}-\mathcal{B} J) .
\end{aligned}
$$

From Theorem 6.2 and Theorem 7.4, we obtain that $r_{\mathbb{R}_{+}}(\mathcal{A}+\mathcal{B} J)$ and $r_{\mathbb{R}_{+}}(\mathcal{A}-\mathcal{B} J)$ are Fredholm operators. Moreover, recalling that $\operatorname{Ker} r_{\mathbb{R}_{+}} \mathcal{K}_{++}=$ $\{0\}$ and $\operatorname{Ker} r_{\mathbb{R}_{+}} \mathcal{K}_{--}=\{0\}$, from identities (6.15), (7.8)-(7.11) and Theorem 7.4 it follows

$$
\begin{aligned}
0 & =\operatorname{Ind} r_{\mathbb{R}_{+}}(\mathcal{A}+\mathcal{B} J)+\operatorname{Ind} r_{\mathbb{R}_{+}}(\mathcal{A}-\mathcal{B} J)=\text { Ind } r_{\mathbb{R}_{+}} \mathcal{K}_{++}+\operatorname{Ind} r_{\mathbb{R}_{+}} \mathcal{K}_{--} \\
& =\left(0-\operatorname{dim} \operatorname{CoKer} r_{\mathbb{R}_{+}} \mathcal{K}_{++}\right)+\left(0-\operatorname{dim} \text { CoKer } r_{\mathbb{R}_{+}} \mathcal{K}_{--}\right) .
\end{aligned}
$$

Thus, we have

$$
\operatorname{dim} \operatorname{CoKer} r_{\mathbb{R}_{+}} \mathcal{K}_{++}=\operatorname{dim} \operatorname{CoKer} r_{\mathbb{R}_{+}} \mathcal{K}_{--}=0
$$

and so we reach to the conclusion that both operators in (5.10) and (5.14) are invertible.

Similarly, we obtain the invertibility results for the operators (5.18) and (5.22). 


\section{Final conclusion}

Due to a direct combination of the results of sections 3-7 and, in particular, Corollary 7.6, we now obtain the main conclusion of the present work for the problems under analysis.

Theorem 8.1. Let $0 \leq \varepsilon<\frac{1}{4}$.

(i) The Problem $\mathcal{P}_{\text {mixed-D }}$ has a unique solution which is representable as a pair $\left(u_{1}, u_{2}\right)$ defined by the formulas (5.5) and (5.6), where the components $\varphi_{0}$ and $-\varphi_{1}$ of the unique solution $\Upsilon$ of the equation (5.7) are used.

(ii) The Problem $\mathcal{P}_{\text {mixed-N }}$ has a unique solution which is representable as a pair $\left(u_{1}, u_{2}\right)$ defined by the formulas (5.11) and (5.12), where the components $\varphi_{0}$ and $-\varphi_{1}$ of the unique solution $\Upsilon$ of the equation (5.13) are used.

(iii) The Problem $\mathcal{P}_{\text {mixed }}$ has a unique solution which is representable as a pair $\left(u_{1}, u_{2}\right)$ defined by the formulas (5.15) and (5.16), where the components $\varphi_{0}$ and $-\varphi_{1}$ of the unique solution $\Upsilon$ of the equation (5.17) are used.

(iv) The Problem $\mathcal{P}_{\text {mixed }}$ has a unique solution which is representable as a pair $\left(u_{1}, u_{2}\right)$ defined by the formulas (5.19) and (5.20), where the components $\varphi_{0}$ and $-\varphi_{1}$ of the unique solution $\Upsilon$ of the equation (5.21) are used.

Moreover all these problems are well-posed (since the resolvent operators are continuous).

To conclude, we would like to point out that although from the natural assumptions in the formulation of the problems (cf. section 2) we were looking for the eventual possibilities for $\varepsilon \in\left[0, \frac{1}{2}\right)$, we now realize that the last result is optimal from the point of view of the possible variability of the Bessel potential spaces smoothness orders (in view to have corresponding well-posed problems); cf. Remark 7.5. It is also worth to mention that this effect is due to the mixed conditions given on the obstacle, while mixed conditions given only on the boundary lead us to the result $\varepsilon \in\left[0, \frac{1}{2}\right)$; cf. [12].

Acknowledgements. L. P. Castro is supported in part by FEDER funds through COMPETE-Operational Programme Factors of Competitiveness 
("Programa Operacional Factores de Competitividade") and by Portuguese funds through the Center for Research and Development in Mathematics and Applications and the Portuguese Foundation for Science and Technology ("FCT-Fundação para a Ciência e a Tecnologia"), within project PEstC/MAT/UI4106/2011 with COMPETE number FCOMP-01-0124-FEDER022690. D. Kapanadze is supported by Shota Rustaveli National Science Foundation within grant FR/6/5-101/12 with the number 31/39.

\section{References}

[1] R.A. Adams, Sobolev Spaces, Academic Press, New York, 1975.

[2] H. Bart, V.E. Tsekanovskii, Matricial coupling and equivalence after extension, Oper. Theory Adv. Appl. 59 (1992), 143-160.

[3] G. Bogveradze, L.P. Castro, On the Fredholm property and index of Wiener-Hopf plus/minus Hankel operators with piecewise almost periodic symbols, Applied Mathematics, Informatics and Mechanics 12(1) (2007), 25-40.

[4] G. Bogveradze, L. P. Castro, On the Fredholm index of matrix WienerHopf plus/minus Hankel operators with semi-almost periodic symbols, Oper. Theory Adv. Appl. 181 (2008), 143-158.

[5] A. Böttcher, Yu.I. Karlovich, I.M. Spitkovsky, Convolution Operators and Factorization of Almost Periodic Matrix Functions, Oper. Theory Adv. Appl. 131, Birkhäuser Verlag, Basel, 2002.

[6] L.P. Castro, D. Kapanadze, On wave diffraction by a half-plane with different face impedances, Math. Methods Appl. Sci. 30 (2007), 513527.

[7] L.P. Castro, D. Kapanadze, Dirichlet-Neumann-impedance boundaryvalue problems arising in rectangular wedge diffraction problems, Proc. Am. Math. Soc. 136 (2008), 2113-2123.

[8] L.P. Castro, D. Kapanadze, Wave diffraction by a strip with first and second kind boundary conditions: the real wave number case, Math. Nachr. 281(10) (2008), 1400-1411. 
[9] L.P. Castro, D. Kapanadze, The impedance boundary-value problem of diffraction by a strip, J. Math. Anal. Appl. 337 (2008), 1031-1040.

[10] L.P. Castro, D. Kapanadze, Exterior wedge diffraction problems with Dirichlet, Neumann and impedance boundary conditions, Acta Appl. Math. 110 (2010), 289-311.

[11] L.P. Castro, D. Kapanadze, Wave diffraction by a half-plane with an obstacle perpendicular to the boundary, J. Differential Equations 254 (2013), 493-510.

[12] L.P. Castro, D. Kapanadze, Mixed boundary value problems of diffraction by a half-plane with a screen/crack perpendicular to the boundary, Proc. A. Razmadze Math. Inst. (2013), to appear.

[13] L.P. Castro, A.S. Silva, Invertibility of matrix Wiener-Hopf plus Hankel operators with symbols producing a positive numerical range, Z. Anal. Anwend. 28(1) (2009), 119-127.

[14] L.P. Castro, A.S. Silva, Fredholm property of matrix Wiener-Hopf plus and minus Hankel operators with semi-almost periodic symbols, Cubo $12(2)$ (2010), 217-234.

[15] L.P. Castro, F.-O. Speck, Regularity properties and generalized inverses of delta-related operators, Z. Anal. Anwend. 17 (1998), 577-598.

[16] L.P. Castro, F.-O. Speck, Relations between convolution type operators on intervals and on the half-line, Integral Equations Oper. Theory $\mathbf{3 7}$ (2000), 169-207.

[17] L.P. Castro, F.-O. Speck, F.S. Teixeira, Explicit solution of a DirichletNeumann wedge diffraction problem with a strip, J. Integral Equations Appl. 5 (2003), 359-383.

[18] L.P. Castro, F.-O. Speck, F.S. Teixeira, On a class of wedge diffraction problems posted by Erhard Meister, Oper. Theory Adv. Appl. 147 (2004), 213-240.

[19] D. Colton, R. Kress, Inverse Acoustic and Electronic Scattering Theory, Springer-Verlag, Berlin, 1998. 
[20] M. Dauge, Elliptic Boundary Value Problems on Corner Domains Smoothness and Asymptotics of Solutions, Lecture Notes in Mathematics 1341, Springer-Verlag Berlin, 1988.

[21] G. Èskin, Boundary Value Problems for Elliptic Pseudodifferential Equations, American Mathematical Society, Providence, Rhode Island, 1981.

[22] G. Hsiao, W.L. Wendland, Boundary Integral Equations, Applied Mathematical Sciences 164, Springer-Verlag, Berlin, 2008.

[23] Yu.I. Karlovich, I.M. Spitkovskiř, Factorization of almost periodic matrix-valued functions and the Noether theory for certain classes of equations of convolution type, Math. USSR Izvestiya 34 (1990), 281316.

[24] P.A. Krutitskii, The Dirichlet problem for the 2-D Helmholtz equation in a multiply connected domain with cuts, Z. Angew. Math. Mech. 77(12) (1997), 883-890.

[25] P.A. Krutitskii, The Neumann problem for the 2-D Helmholtz equation in a multiply connected domain with cuts, Z. Anal. Anwend. 16(2) (1997), 349-361.

[26] P.A. Krutitskii, The Neumann problem for the 2-D Helmholtz equation in a domain, bounded by closed and open curves, Int. J. Math. Math. Sci. 21(2) (1998), 209-216.

[27] P.A. Krutitskii, The 2-dimensional Dirichlet problem in an external domain with cuts, Z. Anal. Anwend. 17(2) (1998), 361-378.

[28] P.A. Krutitskii, The Neumann problem in a 2-D exterior domain with cuts and singularities at the tips, J. Differential Equations 176 (2001), 269-289.

[29] P.A. Krutitskii, The mixed harmonic problem in an exterior cracked domain with Dirichlet condition on cracks, Comput. Math. Appl. 50(56) (2005), 769-782.

[30] P.A. Krutitskii, On the mixed problem for harmonic functions in a 2D exterior cracked domain with Neumann condition on cracks, Quart. Appl. Math. 65(1) (2007), 25-42. 
[31] P.A. Krutitskii, The Helmholtz equation in the exterior of slits in a plane with different impedance boundary conditions on opposite sides of the slits, Quart. Appl. Math. 67(1) (2009), 73-92.

[32] A.I. Komech, N.J. Mauser, A.E. Merzon, On Sommerfeld representation and uniqueness in scattering by wedges, Math. Methods Appl. Sci. 28 (2005), 147-183.

[33] G.D. Malyuzhinets, Excitation, reflection and emission of surface waves from a wedge with given face impedances (English; Russian original), Sov. Phys., Dokl. 3 (1959), 752-755; translation from Dokl. Akad. Nauk SSSR 121 (1959), 436-439.

[34] W. McLean, Strongly Elliptic Systems and Boundary Integral Equations, Cambridge University Press, Cambridge, 2000.

[35] E. Meister, F. Penzel, F.-O. Speck, F.S. Teixeira, Some interior and exterior boundary value problems for the Helmholtz equation in a quadrant, Proc. Roy. Soc. Edinburgh Sect. A 123 (1993), 275-294.

[36] E. Meister, K. Rottbrand, Elastodynamical scattering by N parallel halfplanes in $R^{3}$, Math. Nachr. 177 (1996), 189-232.

[37] E. Meister, F.-O. Speck, A contribution to the quarter-plane problem in diffraction theory, J. Math. Anal. Appl. 130 (1988), 223-236.

[38] E. Meister, F.-O. Speck, Modern Wiener-Hopf methods in diffraction theory. In Ordinary and Partial Differential Equations, Vol. II, B. Sleeman (ed.) et al., Pitman Res. Notes Math. Ser. 216 (1989), 130-171.

[39] A.E. Merzon, F.-O. Speck, T.J. Villalba-Vega, On the weak solution of the Neumann problem for the 2D Helmholtz equation in a convex cone and $H^{s}$ regularity, Math. Methods Appl. Sci. 34 (2011), 24-43.

[40] A. Moura Santos, N.J. Bernardino, Image normalization of Wiener-Hopf operators and boundary-transmission value problems for a junction of two half-planes, J. Math. Anal. Appl. 377 (2011), 274-285.

[41] A. Moura Santos, F.-O. Speck, Sommerfeld diffraction problems with oblique derivatives, Math. Methods Appl. Sci. 20 (1997), 635-652. 
[42] D. Sarason, Toeplitz operators with semi-almost periodic symbols, Duke Math. J. 44 (1977), 357-364.

[43] A. Sommerfeld, Mathematische Theorie der Diffraction (in German), Math. Ann. 47 (1896), 317-374.

[44] A. Sommerfeld, Mathematical Theory of Diffraction (translated from the 1896 German edition and with an introduction by R. J. Nagem, M. Zampolli and G. Sandri; Appendix I by J. Priestley), Progress in Mathematical Physics 35, Birkhäuser, Boston, MA, 2004.

[45] I.N. Vekua, New Methods for Solving Elliptic Equations, John Wiley \& Sons, Inc., New York, 1967 (English translation). 